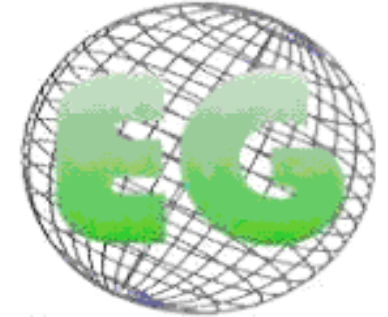

ISSN 1695-6141

$\mathrm{N}^{\circ} 21$

\title{
ALCOHOL Y EMBARAZO: INTERVENCIÓN DE ENFERMERÍA EN ATENCIÓN PRIMARIA
}

\author{
ALCOHOL AND PREGNANCY: NURSING INTERVENTION IN PRIMARY ATTENTION
}

*Martínez Castillo, Antonio

*Enfermero del Área de Gestión Sanitaria Norte de Almería.

Palabras clave: Alcohol; Embarazo; Enfermería; Atención Primaria.

Keywords: Alcohol; pregnancy; infirmary; primary attention

\section{RESUMEN}

La prevalencia de mujeres embarazadas y consumidoras de bebidas alcohólicas varía en función del estudio consultado, pero en todos se muestra con un porcentaje nada despreciable. Las consecuencias de este consumo son muy diversas, pudiéndose producir efectos negativos en cualquier momento de la gestación. Existe un consenso al admitir que no existe una cantidad mínima segura de alcohol que la mujer pueda ingerir, por lo que la recomendación general es que se abstenga durante todo el período prenatal y lactancia. El programa de atención a estas personas tiene como principal objetivo conseguir un control adecuado que repercuta en el bienestar de la madre y el feto. Dicho programa consta de tres elementos claves: identificación/captación, cuantificación de las cantidades ingeridas y seguimiento. Las profesionales responsables son las enfermeras de atención primaria, que dentro del equipo de salud, se muestran imprescindibles para la correcta atención a este colectivo

\section{ABSTRACT}

The prevalence of pregnant women and consumers of alcoholic drinks varies according to the study consulted, but all of them show non negligible percentages. The consequences of this consumption are very diverse, and can produce negative effects at any moment of the gestation. A consensus exists that admits that it there is no minimum safe amount of alcohol that the women can ingest, so the general recommendation is that they refrain throughout the whole prenatal and lactation period. The main objective of the programme of attention to these people is to find a suitable control that focuses on the welfare of the mother and the foetus. Such a programme consists of three key elements: identification/uptake, quantification of the amount ingested and monitoring. The professionals responsible are the nurses of primary attention, who within the health team, are shown to be essential in the correct attention to this collective. 


\section{INTRODUCCIÓN}

El alcohol es la droga que más se consume en España y la que ocasiona más problemas sanitarios y sociales por encima del tabaco y resto de sustancias psicoactivas. (1) De hecho, es considerado como el teratógeno más empleado en el mundo occidental y la principal causa no genética de aparición de defectos congénitos y discapacidad mental. (2)

Durante el año 2003 el 32,9\% de las mujeres de entre 15 y 34 años afirmaba haber consumido alcohol al menos en 8 ocasiones al mes. (3) En el 2005 la prevalencia de borracheras en los últimos 12 meses en mujeres también con edades comprendidas entre 15 y 34 años fue del 20,9\%, cifra que se ha incrementado al 23,2\% en 2007/2008, siendo en este período la media de edad de inicio en el consumo de alcohol en la población en general de 16,8 años (4)

Existe un estudio en el que se estimó que la prevalencia de mujeres embarazadas que consumieron alcohol durante la gestación fue del 55,7\%, un porcentaje que como se puede comprobar no es nada despreciable. Aunque cabe resaltar que dentro de esta cifra se encuentran tanto las mujeres que presentaban un consumo ligero hasta aquellas que lo realizaban de forma excesiva. (5) Sin embargo, existen diferencias en cuanto a estos porcentajes en función del estudio consultado, pues otros autores estiman esta proporción en un $16,1 \%$ (6) o en un $25 \%$. (7)

En este sentido, muchos autores ponen de manifiesto la estrecha vinculación entre los factores socioculturales y el consumo de bebidas alcohólicas durante la gestación. De esta manera, dicho consumo variará en función del nivel de estudios, de la situación laboral, de la edad de la madre, de la ingesta por parte de la pareja, etc. $(2,5)$

Como se muestra más adelante, las consecuencias sobre el embarazo son muchas y de diversa consideración, no estando prácticamente nunca exento de riesgo. Es por esto por lo que la intervención sanitaria está más que justificada.

\section{Efectos del alcohol en el embarazo. Datos que justifican el abordaje enfermero.}

El alcohol atraviesa con facilidad la barrera placentaria, poniéndose rápidamente en contacto con el feto. $(1,8)$ Pero como el cuerpo de éste es inmaduro y no posee las enzimas necesarias para su eliminación, los niveles en su sangre pueden permanecer elevados durante más tiempo que en la sangre materna. (9)

La mayoría de los autores coinciden al afirmar que el riesgo de la ingesta de alcohol existe durante todo el embarazo, pudiéndose producir daños tanto en la organogénesis como en la fase madurativa. (2) De esta forma, las consecuencias variarán según la edad gestacional. Como norma general, las malformaciones se producen en el período embrionario y los defectos neurológicos y el crecimiento retardado ya en el período fetal. (8)

Con respecto al riesgo derivado del consumo moderado u ocasional existen disparidad de opiniones pues los resultados obtenidos en diferentes estudios concluyen ideas contrapuestas. Martínez-Frías M. (2) refiere, aunque con cierta cautela, que consumir pequeñas cantidades de alcohol no aumenta el riesgo de sufrir daños durante el embarazo. Estos mismos autores, en una publicación posterior, concluyeron que con dosis bajas se incrementa el riesgo de daños oculares y de anomalías faciales (10) También, en un metaanálisis se comprobó que el consumo moderado, considerando éste de 2 a 14 bebidas o de 28 a $196 \mathrm{~g}$. de alcohol a la semana, no aumentaba el riesgo de malformaciones prenatales (11) y en un estudio de cohortes llevado a cabo en Valencia no se encontró una 
relación significativa entre el consumo de alcohol a dosis bajas o moderadas y el bajo peso del niño al nacer. (12) En este mismo sentido, encontramos otro autor que concluye que el consumo bajo o moderado en el inicio del embarazo no afecta al crecimiento del feto (12)

Sin embargo, cuando se habla de una ingesta mayor de bebidas alcohólicas durante la gestación, la mayoría de los autores comparten posturas y opiniones en cuanto al riesgo que supone para el embarazo. Además, un hecho probado es que existe una relación directa entre la dosis de alcohol consumida y el riesgo de defectos congénitos y otros daños sobre el feto, sin ir más lejos, la edad gestacional y el bajo peso al nacer disminuyen conforme se aumenta la cantidad ingerida. $(13,14)$

En un estudio llevado a cabo en España, se observó que entre las madres que consumieron más de $500 \mathrm{ml}$. de vino más alguna copa al día o aquellas que presentaba un consumo crónico, era más probable que los hijos nacieran con malformaciones que aquellas madres con ingestas menores. (2) También se puede afirmar que aumenta el riesgo de abortos espontáneos y de nacimiento de niños muertos. En este sentido, Kesmodel U. et al, (15) en una investigación llevada a cabo en Dinamarca pusieron de manifiesto que las mujeres que consumieron 5 o más bebidas alcohólica a la semana tienen tres veces más probabilidades de que el niño nazca sin vida que las que consumieron menos de una bebida a la semana.

En los casos más graves, como pueden ser aquellas mujeres con un consumo crónico, (16) los daños se manifiestan por el conocido síndrome alcohólico fetal, caracterizado principalmente por un retraso en el crecimiento pre y postnatal, alteraciones del sistema nervioso central, alteraciones cráneo-faciales y en algunos casos también aparecen lesiones y malformaciones en otros órganos y aparatos.

Por otro lado, también se pueden encontrar evidencias científicas que muestran consecuencias producidas a largo plazo: En un estudio publicado en el año 2002 se comprobó que los niños de 14 años cuyas madres consumieron una sola bebida alcohólica a la semana a lo largo de la gestación, eran más bajos y delgados y además presentaban un perímetro craneal más pequeño que los hijos de madres que consumieron menos de una bebida. (17) En otra publicación se concluye que los niños de 6 y 7 años de edad y de madres que habían ingerido solo una bebida semanalmente mientras estaban embarazadas, presentaban un aumento notable de la agresividad, medida ésta mediante una escala validada. (14) Ante estos resultados es interesante recordar la controversia mencionada anteriormente respecto al consumo ocasional y moderado de alcohol, lo que nos puede llevar a una profunda reflexión al respecto; vemos que con consumos mínimos (1 bebida a la semana) se pueden producir efectos negativos aunque sean a largo plazo.

Ante todos estos datos, y de acuerdo con la mayoría de los autores, se puede afirmar que no existe una cantidad segura de alcohol consumido por la mujer gestante, por lo que las recomendaciones formuladas por los profesionales sanitarios deben ir encaminadas a evitar la ingestión de cualquier cantidad de alcohol mientras dure el embarazo y la lactancia. (11)

\section{Objetivos del programa de intervención enfermera:}

- Lograr un mejor control de la mujer embarazada consumidora de alcohol.

- Conseguir la abstinencia total o en su defecto la disminución de la cantidad de alcohol consumido. 


\section{MATERIAL Y MÉTODO}

La atención enfermera consta de tres fases interconectadas: Identificación/captación de gestantes bebedoras de alcohol, cuantificación de la cantidad ingerida y seguimiento.

\section{FASE 1: Identificación /captación.}

Como es evidente, el primer paso a seguir es reconocer a todas las mujeres embarazadas y que realizan un consumo de alcohol, sea del tipo que sea, así como a todos los factores de riesgo asociados. Este paso no siempre puede resultar sencillo, ya que existen aquellas gestantes que niegan su consumo, por lo que pasarán desapercibidas y no serán incluidas en el programa. También están las que reconociendo la ingesta de alcohol, afirman beber cantidades menores a las que en realidad beben, teniendo de esta forma un control inferior al que precisarían tener.

Todos los miembros del equipo sanitario tienen la responsabilidad de identificar a estas mujeres, pero la enfermería, por sus características, ocupa un papel importante en este ámbito.

Para la captación, el profesional cuenta con varios instrumentos que se resumen a continuación:

- Entrevista personal: Es un elemento básico en toda actuación sanitaria y prácticamente la única manera de reconocer a aquellas mujeres que beben de forma esporádica o moderada, ya que los instrumentos que se detallan más adelante pueden sólo ser válidos para las que presentan un consumo más importante. Debe tenerse en cuenta el grado de participación por parte de la mujer, ya que de ello dependerá la validez de la entrevista realizada. Como ya se ha comentado, la ocultación del problema y su negación representan un sesgo importante. En la entrevista, pueden ser de utilidad los cuestionarios validados. Entre los más utilizados se encuentran el CAGE camuflado para la identificación del consumo excesivo de alcohol, o el MALT para el diagnóstico de alcoholismo. Ambos cuestionarios presentan una alta sensibilidad y especificidad. (18)

- Historia clínica: La consulta de la historia clínica de la mujer ha de ser también un paso ineludible. En ella se pueden encontrar datos de interés como diagnósticos anteriores de consumo excesivo de alcohol, de episodios de embriaguez, que aunque no son concluyentes, sí deben ser tenidos en cuenta, etc.

- Marcadores bioquímicos: El análisis de alcohol en sangre u orina no siempre resulta de utilidad en una consulta de atención primaria, ya que la duración de éste en los mencionados fluidos corporales es de tan solo unas horas. Es por esto por lo que se utilizan otros marcadores bioquímicos. La limitación que presentan es la baja sensibilidad y especificidad de todos ellos por sí mismos a la hora de determinar un consumo de alcohol. El más representativo de los marcadores es la gammaglutamiltranspeptidasa (GGT) seguido del volumen corpuscular medio (VCM) cuya elevación puede ser señal de un consumo crónico. También pueden considerarse de utilidad la elevación del coeficiente glutámico-oxalacético, transaminasa/glutámico-pirúvico, transaminasa (GOT/GPT), los triglicéridos y el ácido úrico. $(18,19)$ La interpretación de estos marcadores debe ir acompañada de los datos aportados por los demás 
instrumentos que se exponen en el presente apartado, como la entrevista o el examen físico.

- Examen físico: Signos tales como el enrojecimiento de la piel de la cara y las conjuntivas y un temblor fino en boca y manos, pueden ser sospecha de una ingesta importante de alcohol.

\section{FASE 2: Cuantificación de la cantidad ingerida.}

Una vez identificada la mujer gestante consumidora de alcohol es necesario establecer la cantidad aproximada que es ingerida en un período de tiempo determinado. Para ello se empleará el sistema propuesto por la Sociedad Española de Medicina de Familia y Comunitaria que se basa en la medición por unidades tal y como se muestra en la Tabla 1.

\begin{tabular}{|c|c|c|}
\hline \multicolumn{3}{|c|}{ TABLA 1. CÁLCULO DEL CONSUMO DE ALCOHOL EN UNIDADES } \\
\hline TIPO DE BEBIDA & VOLUMEN & UNIDADES \\
\hline \multirow[t]{2}{*}{ Vino } & 1 vaso $(100 \mathrm{cc})$ & 1 \\
\hline & 1 litro & 10 \\
\hline \multirow[t]{2}{*}{ Cerveza } & 1 caña $(200 \mathrm{cc})$ & 1 \\
\hline & 1 litro & 5 \\
\hline \multirow[t]{3}{*}{ Whisky, ron, coñac,... } & 1 copa $(50 \mathrm{cc})$ & 2 \\
\hline & 1 combinado $(50 \mathrm{cc})$ & 2 \\
\hline & 1 litro & 40 \\
\hline \multirow[t]{2}{*}{ Jerez, vermut, cava,... } & 1 copa $(50 \mathrm{cc})$ & 1 \\
\hline & 1 litro & 20 \\
\hline \multicolumn{3}{|c|}{$\begin{array}{l}\text { De: Robledo T, Córdoba R. Cómo actuar ante el consumo de alcohol: Guía de referencia } \\
\text { para profesionales de atención primaria. Barcelona: Sociedad Española de Medicina de } \\
\qquad \text { Familia y Comunitaria: } 2^{a} \text { Ed., } 2007 .\end{array}$} \\
\hline
\end{tabular}

El hecho de conocer la cantidad y frecuencia de consumo es básicamente orientativo, pues como se ha indicado previamente, no existe una cantidad segura de alcohol que pueda beber una embarazada, cualquier cantidad puede suponer un riesgo. De esta forma toda mujer que afirme tomar bebidas alcohólicas durante el embarazo, independientemente de la cantidad y la frecuencia, debe considerarse de riesgo hasta nuevas valoraciones en sucesivas visitas en las que se confirme la continuidad o la supresión de este hábito. Pero la cantidad ingerida nos orientará a la hora de determinar el número de visitas que debe hacer la paciente. Así, a más cantidad de alcohol más número de visitas y mayor control sanitario. 
Es importante tener en cuenta en esta parte la minimización por parte de las madres a la hora de cuantificar las unidades de bebida, lo que puede llevar al profesional a infravalorar el problema.

\section{FASE 3: Seguimiento de la gestante.}

Con un adecuado seguimiento de la mujer gestante identificada previamente como consumidora de bebidas alcohólicas se debe garantizar un aumento del bienestar y calidad de vida de ésta y de su futuro hijo.

El seguimiento de la mujer se realizará en función de las características de la misma y de la cantidad de alcohol que ingiera.

El personal de enfermería debe tener en cuenta que el seguimiento puede ser muy complicado en grandes bebedoras y en mujeres con problemas sociales relacionados, por lo que estas personas requieren de un esfuerzo añadido para lograr el mantenimiento dentro del programa; es por esto también por lo que las visitas han de adaptarse a su situación, intentando que existan el mínimo de interferencias. Para esto habrá que facilitar los horarios, disminuir los trámites burocráticos, colaborar en los trámites administrativos, etc.

Es importante que la mujer perciba la intervención sanitaria como una ayuda a su problema y no como una limitación. Para ello, se debe transmitir una sensación de comprensión e interés por la salud y calidad de vida de la madre y el feto, procurando evitar acciones que puedan ser interpretadas de forma negativa, tales como el enjuiciamiento de la situación de la mujer.

El control de la gestante debe extenderse al postparto durante el tiempo que se estime necesario, planteando las mismas metas que durante el embarazo, pero realizando a su vez una vigilancia detallada del estado de salud del recién nacido.

Las principales áreas de actuación incluidas en el seguimiento de la gestante consumidora de alcohol son las siguientes:

- Ofrecer información y escuchar: Las recomendaciones deben ser sencillas y claras, de fácil comprensión y adaptadas al nivel intelectual de la paciente. La información debe contener tanto los riesgos que supone el consumo de alcohol como los beneficios de su disminución.(19) Además de ofrecer datos y recomendaciones, es necesario resaltar la importancia de la escucha activa por parte de la enfermera, que ha de favorecer a su vez un ambiente adecuado para que la mujer pueda expresarse con libertad de manera que exista un diálogo fluido.

- Identificación y control de factores de riesgo: Es necesario conocer los factores de riesgo asociados al consumo de alcohol. Es posible que la persona presente otros hábitos que junto con la ingesta de bebidas alcohólicas representen un factor de riesgo para la aparición de complicaciones. Además, el profesional debe indagar en aquellos posibles factores que provocan o incrementan que la mujer beba a lo largo de la gestación, tales como estrés, depresión, malos tratos, problemas laborales, etc. Identificarlos y trabajar sobre ellos es imprescindible para un correcto seguimiento y una adecuada atención. 
- Identificación y control de complicaciones: Mediante un exhaustivo seguimiento se pueden evitar complicaciones en el embarazo que supongan un riesgo para el bienestar de la madre y el feto. Pero también se pueden identificar de forma precoz aquellas complicaciones que ya hayan aparecido, de manera que permita actuar sobre ellas lo más prontamente posible y favoreciendo la derivación en caso necesario a otros profesionales. De esta forma, si la enfermera detecta algún problema podrá solucionarlo precozmente si está dentro de sus posibilidades, o bien podrá derivar a la mujer a su médico de familia y éste a su vez al especialista correspondiente si fuera necesario.

- Monitorización del consumo: Ayuda al profesional a evaluar la disminución de la ingesta. La cuantificación de las cantidades ingeridas se hará en cada visita mediante la entrevista utilizando el sistema de unidades (tabla 1). Además, se pueden monitorizar los marcadores bioquímicos (GGT, VCM, GOT) que junto con la entrevista sirven de indicadores en la evolución del consumo. Es importante la negociación con la mujer a la hora de determinar la disminución, de forma que los objetivos marcados en este sentido se hagan de forma consensuada y no de forma impuesta.

- Apoyo sanitario, psicológico y social: La enfermera debe representar un apoyo en todos estos aspectos sin menospreciar ninguno, pues es posible que la clave del éxito del programa se encuentre dentro de alguno de ellos. Como se ha expuesto, cabe la posibilidad de que el consumo de alcohol esté condicionado por algún factor psicológico o social. La derivación a otros profesionales (médicos de familia, ginecólogos, psicólogos, trabajadores sociales, equipo de drogodependencias, etc.) no exime a la enfermería de la responsabilidad de un adecuado seguimiento.

\section{RESULTADOS ESPERADOS TRAS LA INTERVENCIÓN}

La implantación de un programa de intervención enfermera en el ámbito de la Atención Primaria de la Salud a mujeres gestantes consumidoras de alcohol debe ofrecer los siguientes resultados:

- Prevención de las posibles complicaciones que pueden aparecer a lo largo del embarazo.

- Actuación precoz sobre complicaciones ya aparecidas.

- Las mujeres que realizaban un consumo moderado-bajo de alcohol debido a creencias erróneas sobre los efectos del mismo (como son aquellas que tomaban una copa de vino durante el almuerzo) dejan de beber.

- Aquellas mujeres que realizaban consumos elevados, tras la intervención, también consiguen abstenerse de ingerir cualquier cantidad.

- Las gestantes con consumos elevados que no logran dejar de beber durante el embarazo, disminuyen considerablemente las cantidades ingeridas, con el consecuente beneficio. 
- Como consecuencia de los puntos anteriores, se eliminan o disminuyen los posibles efectos negativos que el feto y la madre puedan sufrir.

\section{DISCUSIÓN Y CONCLUSIONES}

El objetivo principal de este programa es lograr un mejor control de la mujer embarazada consumidora de alcohol, de esta forma conseguiremos por una parte el aumento del bienestar y la calidad de vida de la gestante y por otra, la del feto. A su vez, el programa plantea un segundo objetivo: conseguir la abstinencia total o en su defecto la disminución de la cantidad de alcohol consumido. Como se puede observar, el principal objetivo no es conseguir la abstinencia, aunque también este aspecto forme parte importante del programa. Esto se debe a que en muchos casos, como en grandes bebedoras o en personas que conviven en un entorno donde el consumo de alcohol de forma moderada en el embarazo está asumido, conseguir el abandono total será más que difícil. Por ello, aparte de insistir en la disminución del consumo, la intervención por parte del personal de enfermería, sustenta sus bases en el aumento del control para evitar en la medida de lo posible la aparición de complicaciones.

Las enfermeras son, en muchos casos, la puerta de entrada al sistema sanitario por la cercanía con la que son percibidas por parte del usuario. Es por ello por lo que pueden considerarse como la base a partir de la cual se desencadenan una serie de actuaciones con el fin de mejorar la calidad de vida de las gestantes y sus futuros hijos.

Además, la enfermería de Atención Primaria se muestra como un elemento clave de esta intervención, pues es en este medio donde se dan las condiciones idóneas para que estos profesionales puedan llevar a cabo un seguimiento estrecho con las mujeres gestantes en general y con aquellas que sean consumidoras de alcohol en particular.

Para ello siempre será necesario propiciar y facilitar la adquisición de conocimientos y las habilidades necesarias para que la enfermería actúe de manera coherente con la evidencia científica acerca del tema. También será de gran utilidad contar con un programa estructurado que marque las pautas elementales de la atención enfermera.

\section{BIBLIOGRAFÍA}

1. Ministerio de Sanidad y Consumo. Guía sobre drogas. Madrid: Delegación del Gobierno para el Plan Nacional sobre Drogas; 2007.

2. Martínez-Frías ML, Bermejo E, Rodríguez-Pinilla E. Evolución temporal y por comunidades autónomas del consumo de diferentes cantidades de alcohol durante el embarazo. Med Clin 2003; 120(14): 535-541.

3. Ministerio de Sanidad y Consumo. Informe 2004 del Observatorio Nacional sobre Drogas. Madrid: 2004.

4. Ministerio de Sanidad y Consumo. Informe de la encuesta domiciliaria sobre alcohol y drogas en España (Edades) 2007/2008. Disponible en: URL: http://www.pnsd.msc.es/Categoria2/observa/estudios/home.htm

5. Eiman $\mathrm{M}$, et al. Estudio sobre consumo de alcohol durante el embarazo. Área de Salud V de la Comunidad de Madrid. Boletín Epidemiológico Semanal. Instituto de Salud Carlos III 1997; 32(5): 301-312. 
6. Castillo R. Consumo de alcohol en mujeres embarazadas. Enferm Comunitaria 2008; $4(1)$.

7. Díez T. Encuesta alcohol y gestación. Enferm Científ 1997; 178-179:14-19.

8. Comisión Clínica de la delegación para el Plan Nacional sobre Drogas. Informe sobre alcohol. Madrid: Ministerio de Sanidad y Consumo; 2007

9. Cancino F, Zegarra J. Síndrome alcohólico fetal. Revista de Neuro-Psiquiatría 2003; 66: 302-312.

10. Martínez-Frías M, Bermejo E, Rodríguez -Pinilla E, Fría JL. Risk for congenital anomalies associated with different sporadic and daily doses of alcohol consumption during pregnancy: a case-control study. Birth Defects Res A Clin Mol Teratol 2004; 70:194-200.

11. Polygenis $\mathrm{D}$, et al. Moderate alcohol consumption during pregnancy and the incidence of fetal malformations: A meta-analysis. Neurotoxicol Teratol 1998; 20:61-67.

12. Bolumar $\mathrm{F}$, et al. Efecto del consumo moderado de alcohol durante el embarazo sobre el peso del recién nacido. Med Clin 1994; 102(20): 765-768.

13. Aguilera C, Izarra A. Abuso de sustancias tóxicas durante el embarazo. Med Clin 2005; 125(18):714-716.

14. Sood B, Delayne-Black V, et al. Prenatal alcohol exposure and childhood behavior at age 6 to 7 years: I. Dose-response effect. Pediatrics 2001; 108:34-42.

15. Kesmodel $\mathrm{U}$, et al. Moderate alcohol Intake during pregnancy and the risk of stillbirth and death in the firs year of live. American Journal of Epidemiology 2002; 155(4):305-312.

16. Parés A, Caballería J. Patología orgánica. Adicciones 2002; 14: 155-173.

17. Day N, et al. Prenatal alcohol exposure predicts continued deficits in offspring size at 14 years of age. Alcohol Clin Exp Res 2002; 26(10): 1584-1591.

18. Bruguera M, Guai A, Salleras L, Rodés J. Cribado del consumo excesivo de alcohol. Med Clin 1994; 102 supl 1: 85-92.

19. Robledo T, Córdoba R. Cómo actuar ante el consumo de alcohol: Guía de referencia para profesionales de atención primaria. Barcelona: Sociedad Española de Medicina de Familia y Comunitaria: $2^{\mathrm{a}}$ Ed., 2007. 